\title{
Research on medical ultrasound image processing
}

\author{
Wang Jinguo ${ }^{1,}$, Wang $\mathrm{Na}^{2, \mathrm{~b}^{*}}$ (corresponding author), Wang rui ${ }^{3, \mathrm{c}}$ \\ ${ }^{1}$ Department of Urology, the First Hospital of Jilin University, China \\ ${ }^{2}$ Department of Anesthesiology, the First Hospital of Jilin University, China \\ ${ }^{3}$ Department of Anesthesiology, the First Hospital of Jilin University, China \\ âwangjinguolily@163.com, ${ }^{\mathrm{b}}$ lilyly12345@163.com, ${ }^{\mathrm{C} X i a o b e n 6666 @ 126 . c o m ~}$
}

Keywords: Medical ultrasound image. Brightness Mode Ultrasonic. image enhancement.

Abstract. Image processing plays an important role in the quantitative and qualitative analysis of medical ultrasound images. It has a direct impact on the follow-up analysis. The correct processing result is the guarantee for the clinical application of the diagnostic information from the ultrasonic image, and it is also the important part of the quantitative analysis and the real-time monitoring and accurate positioning in the clinical.

\section{Introduction}

Medical imaging has become one of the most rapidly developing areas in the medical technology, which makes it more direct and clear to observe the internal pathological changes of human body. In recent years, with the advent of the information age, especially the digital age, computed tomography, magnetic resonance imaging, ultrasonography new medical imaging technology has been widely used in medical diagnosis, preoperative planning, treatment, postoperative monitoring and other aspects[1][2].

The purpose of these imaging techniques is to obtain a comprehensive and accurate quantitative data for patients, and provide the correct digital information for the diagnosis, treatment planning, operation and postoperative assessment[3]. They will lead to a new revolution in the field of medical imaging. Computer and medical image processing technology, which is the basis of the development of these imaging techniques, is leading to a profound transformation in the modern medical diagnosis[4]. The clinical applications of various new medical imaging methods, which make the medical diagnosis and treatment technology has made great progress, and the information obtained by various imaging techniques are complementary, and also provide a powerful scientific basis for clinical diagnosis and biomedical research. Therefore, medical image processing technology has been highly valued by domestic and foreign experts[5].

In the field of medical imaging, ultrasonic imaging technology has played a huge role in its unique advantages, and has been widely used in clinic.

First of all, it is a non-invasive method for non-invasive examination. Compared with X-ray, CT scan and magnetic resonance imaging, ultrasound imaging has no radiation to the human body, and it is the most safe when repeated exposure to the standard energy[6].

Secondly, the ultrasonic imaging is fast and has the characteristics of real-time. Nuclear magnetic resonance, X-ray, CT scan to obtain data for a long time, there is a more complex postprocessing process, the imaging speed is slow.

Moreover, the ultrasonic imaging equipment is simple, the cost is low, the operation is convenient, easy to use.

At present, it is difficult to compare with MRI and CT in automatic and semi - automatic 
quantitative analysis. However, ultrasonic imaging has great potential in the field of quantitative analysis, real-time monitoring and treatment planning.

\section{Ultrasonic principle}

Ultrasound is than human auditory range of sound waves, usually refers to the frequencies above $20 \mathrm{kHz}$ high frequency vibrator detection, ultrasonic frequency of application in medical diagnosis in general between $1 \mathrm{MHz}$ to tens of $\mathrm{MHz}$.

The important role of ultrasound in medicine is that it can not only penetrate the human body, but also interact with the body tissue. Ultrasonic diagnosis and treatment in clinical applications are divided into two aspects: ultrasonic diagnosis is a high frequency and low intensity ultrasound, high frequency can improve the resolution of the organization, in order to get a clear, detailed image, while the low voice can reduce the side effects of tissue damage. Ultrasonic therapy is a low frequency and high sound intensity of ultrasound, low frequency ultrasound to increase the penetration rate of the tissue, while the loud (especially focused) ultrasound can be used to organize the production: biological effects, for selective destruction of focal lesions.

\section{Brightness Mode Ultrasonic and imaging principle}

Brightness mode ultrasonic imaging is the basic principle is: to the human body to launch a group of ultrasound, in a certain direction to scan. According to the delay time and the intensity of monitoring the echo, the distance and the nature of the organs can be judged. After electronic circuits and computer processing, we have formed the brightness mode ultrasonic images of the images we have seen. The key part is the brightness mode ultrasonic probe, which has a set of ultrasonic transducers, which is made of a set of special crystals with piezoelectric effect. The piezoelectric crystal with special properties, is in crystal in a specific direction and voltage, the crystals will deform, in turn when the deformation of the crystal, corresponding to the direction will generates a voltage to achieve the conversion of electrical signals and ultrasonic.

Brightness mode ultrasonic imaging of the work process is: when the probe to obtain excitation pulse after the launch of ultrasonic, and then after a period of time after receiving the echo signal from the probe, the probe received echo signal filtering, logarithmic amplification and other signal processing, and then by the DCS circuit to form digital signal, in the CUP control to further image processing, and measurement circuit together with the synthesis of video signal to form our familiar images, also known as two-dimensional black and white ultrasound image.

\section{Ultrasonic image enhancement and noise suppression}

For ultrasonic image processing system, the process can be divided into three stages, in the acquisition of the original image, the first is the image preprocessing stage, second is the feature extraction stage, the third is the identification analysis stage. In practical application, the image quality is not high because of the noise, illumination and so on. So the image quality is not high, so we need to carry on the pretreatment to extract the information we are interested in. Image preprocessing includes image enhancement, smoothing filtering, sharpening, noise removal, etc.. Image preprocessing can be realized in the spatial domain, and it can be realized in the frequency domain. We mainly introduce the image enhancement and noise suppression in the spatial domain. Ultrasonic image enhancement. At present, there are two kinds of enhancement techniques, which 
can be divided into the image based method and the method based on the transform domain. As the name implies, the former is directly processed in the image space, and the image processing is carried out indirectly by the transform domain of the image. The spatial domain technique is based on the gray level mapping transform; the fundamental of the frequency domain is the convolution theorem. In the process of image processing, the space is the space of the pixels. Spatial enhancement method is a direct effect on the enhancement method, which can be expressed as:

$$
g(x, y)=E H[f(x, y)]
$$

In which $f$ and g were enhanced the image before and after, while the $\mathrm{EH}$ is enhanced the operation.

If $\mathrm{EH}$ is defined on each point (x, y), then $\mathrm{EH}$ is a point operation, also known as the gray transform. If $\mathrm{EH}$ is defined in a certain field of $(\mathrm{x}, \mathrm{y})$, the $\mathrm{EH}$ is called a template operation, also known as spatial filtering.

Ultrasonic image noise suppression. Due to the limitation of imaging system, the image is often affected by various noise sources in the process of generation and transmission. The image quality is relatively poor, which has been the main shortcoming of ultrasonic image, especially because of the in-homogeneity of the imaging organ or tissue structure. Some small structures can not be resolved by ultrasonic, and the interference of acoustic signal is formed.

Speckle noise seriously affects the quality of the image, which has brought difficulties to the medical diagnosis and automatic recognition, which makes it more difficult to identify and analyze the image details. Reflected in the image, the noise makes the original uniform and continuous variation of the gray suddenly become big or reduced, forming a number of false edges or contours of objects.

With the appearance of some new ultrasound imaging technology, the quality of the image is improved, but the quality of the image still has a big gap with the requirements of image analysis and image reconstruction. Image enhancement, suppression or elimination of noise is one of the necessary preparations for image segmentation. Especially for ultrasound image, in order to improve the pathological features, we must carry out the image smoothing filter, we mainly consider the spatial domain.

Because of the ultrasonic image, the research work of the ultrasonic image filtering algorithm is mainly focused on the suppression of its speckle noise. Studies have shown that ultrasonic image in the original spot signal with the Rayleigh distribution characteristics, is proportional to its mean and standard deviation, can be regarded as independent of the image signal and the multiplicative noise, and the signal processing of speckle statistical characteristic of signals is changed, its mean and variance proportional.

When each resolution element (by the beam width and the receiver decision) scattering in Yuan is large, the noise signal obeys the Rayleigh distribution:

$$
P(x)=\left(x^{2} / \sigma\right) \exp \left[-x^{2} /\left(2 \sigma^{2}\right)\right] \quad x>=0
$$

In which, $x$ is scattering intensity. $\sigma^{2}$ is the variance of the scattering intensity of each scattering unit. When the scattering elements are less, the noise signals obey the $\mathrm{K}$ distribution.

$$
P(x)=\left[2 b /(\Gamma(\alpha)](b x / 2)^{\alpha} K_{\alpha-1}(b x)\right.
$$


In which, $b=2\left[\alpha / E\left(x^{2}\right)\right]^{1 / 2} \quad(x>=0)$, a $>0$ is a characteristic parameter for describing the scattering density of $\mathrm{K}$ distribution. $\mathrm{K}$ is the modified Bessel function. $\Gamma$ is the Gamma function. $E\left(x^{2}\right)$ is the two order moment of $x$.

In ultrasonic images, the dynamic range of echo signal is very large, and the dynamic range is very small, so it is necessary to do a logarithmic compression of the echo signal, thus changing the original statistical characteristics of the echo signal.

\section{References}

[1] Symon D O.Colour, colour spaces and the human visual system. Technical report, School of Computer Science, University of Birmingham, England . 1989.

[2] Uchiyama T,Arbib M A.colour image segmentation using competitive learning. IEEE Transactions on Pattern Analysis and Machine Intelligence . 1994.

[3] Gudrun J. Klinker,Steven A. Shafer,Takeo Kanade. A physical approach to color image understanding[J]. International Journal of Computer Vision . 1990 (1).

[4] Michael Kass,Andrew Witkin,Demetri Terzopoulos. Snakes: Active contour models[J]. International Journal of Computer Vision . 1988 (4).

[5] Kim V. and Yaroslavskii L.Rank algorithms for picture processing. Computer Vision . 1986.

[6] S.Zeki.Colour coding in the cerebral cortex: The reaction of cells in monkey visual cortex to wavelengths and eolours. Neuroscience. 1983. 